\title{
Transplantation of Autologous Bone Marrow Mesenchymal Stem Cells into the Testes of Infertile Male Rats and New Germ Cell Formation
}

\author{
Mohammad Ghasemzadeh-Hasankolaei ${ }^{1}$, Roozali Batavani ${ }^{2}$, \\ Mohamadreza Baghaban Eslaminejad ${ }^{3}$, Foroughazam Sayahpour ${ }^{3}$ \\ ${ }^{I}$ Infertility and Reproductive Health Research Center, Health Research Institute, Babol University of Medical Sciences, Babol, Iran, \\ ${ }^{2}$ Department of Clinical Sciences, Faculty of Veterinary Medicine, Urmia University, Urmia, Iran, \\ ${ }^{3}$ Department of Stem Cells and Developmental Biology, Cell Science Research Center, \\ Royan Institute for Stem Cell Biology and Technology, ACECR, Tehran, Iran
}

Background: Mesenchymal stem cells (MSCs), have been suggested as a potential choice for treatment of male infertility. Yet, the effects of MSCs on regeneration of germinal epithelium of seminiferous tubules and recovery of spermatogenesis have remained controversial. In this research, we have evaluated and compared the fate of autologous bone marrow (BM)-MSCs during three different periods of time- 4, 6 and 8 weeks after transplantation into the testes of busulfan-induced infertile male rats.

Methods: Rats BM samples were collected from tibia bone under anesthesia. The samples were directly cultured in culture medium. Isolated, characterized and purified BM-MSCs were labeled with PKH26, and transplanted into the testes of infertile rats. After 4, 6 and 8 weeks, the testes were removed and underwent histological evaluations. Results: Immunohistochemical analysis showed that transplanted BM-MSCs survived in all three groups. Some of the cells homed at the germinal epithelium and expressed spermatogonia markers (Dazl and Stella). The number of homed spermatogonia-like cells in 4-week testes, was more than the 6-week testes. The 8-week testes had the least numbers of homed cells $(p<0.05)$. Immunostaining for vimentin showed that BM-MSCs did not differentiate into the sertoli cells in the testes. Conclusions: From our results, it could be concluded that, autologous BM-MSCs could survive in the testis, migrate onto the seminiferous tubules basement membrane and differentiate into spermatogonia. Although, no more differentiation was observed in the produced spermatogonia, generation of such endogenous GCs would be a really promising achievement for treatment of male infertility using autologous stem cells.

Keywords: Autologous, Bone marrow mesenchymal stem cells, Infertile male rats, Transplantation, Testis, Germ cells

Accepted for publication April 19, 2016, Published online November 30, 2016

Correspondence to Mohammad Ghasemzadeh-Hasankolaei

Stem Cell Research Lab., Infertility and Reproductive Health Research Center, Health Research Institute, Babol University of Medical Sciences, Amirkola, Babol 47135-547, Iran

Tel: +98(11)32354327-8, Fax: +98(11)32354328

E-mail: ghasemzadeh78@gmail.com, m.ghasemzadeh@mubabol.ac.ir

Co-Correspondence to Roozali Batavani

Department of Clinical Sciences, Faculty of Veterinary Medicine, Urmia University, Serow Highway, Urmia 1177, Iran

Tel: +98(44)32774737, Fax: +98(44)32777099

E-mail: alibatavani@yahoo.com

Co-Correspondence to Mohamadreza Baghaban Eslaminejad

Royan Institute, Bani Hashem Sq., Bani Hashem St., Resalat Highway, Tehran 19395-4644, Iran

Tel: +98(21)22307960-6, Fax: +98(21)22310406

E-mail: eslami@royaninstitute.org

() This is an open-access article distributed under the terms of the Creative Commons Attribution Non-Commercial License (http://creativecommons.org/ licenses/by-nc/4.0/), which permits unrestricted non-commercial use, distribution, and reproduction in any medium, provided the original work is properly cited. 


\section{Introduction}

Male infertility is one of the most important issues that affect the personal and social life of a family and its members. Numerous causes are responsible for male infertility, for example environmental factors include infections, smoking, heavy metal exposure, radiation, increase in scrotal temperature and certain drugs, in addition to anatomical defects such as absence of a part of the vas deferens and epididymal obstructions, endocrinopathies that include sex hormone imbalances or lack of secretion of certain hormones (GnRH, FSH, LH), ejaculatory failures (disorders in the dorsal penile nerve), genetic factors [disorders in the Y chromosome and/or in the genes involved in the evolution of germ cells (GCs)] and disorders in proliferation and differentiation of GCs (1-3). Common medical treatments for male infertility consist of surgery (4), hormone and drug therapies (2). In recent years, after identifying the high capability of stem cells to produce different cell types, a number of scientists have proposed the use of stem cells and cell-based therapies as a possible new therapeutic choice for male infertility (5). Stem cells are undifferentiated cell types that have two main characteristics - self-renewal with the production of identical daughter cells and the ability to differentiate into more specialized cell types. The two main stem cells classes are embryonic and adult stem cells (5).

Mesenchymal stem cells (MSCs) are a group of multi/pluripotent adult stem cells that reside in almost all tissues, including bone marrow (BM), lungs, liver, umbilical cord blood, umbilical cord Wharton's jelly, peripheral blood, eyes, skin, adipose tissue, amniotic fluid, pancreas, and skeletal muscles (6-8). MSCs are of interest because they can be easily isolated from a small primary tissue sample such as BM, fat tissue, blood samples, and amniotic fluid. They are highly proliferative, colonogenic cells $(9,10)$. It has been shown that these cells have the potential to differentiate into different mesodermal and non-mesodermal cells and germline cell types (6, 7, 11-13). Scientists suggest that MSCs are good candidates for regenerative medicine and cell-based therapies for tissue and organ injuries, and for chronic diseases (14-17). This high differentiation capacity has attracted scientists to study the possibility of MSCs as treatment for male infertility. Disruptions to GC proliferation and differentiation are one of the most important causes of male infertility (1), thus in vitro derivation of GCs or regeneration of a native GC pool are major topics for infertility studies. A number of research groups have focused on in vitro derivation of GCs from MSCs, whereas others focused on regeneration of the germinal epithelium of the testis by transplantation of MSCs. Promising achievements have been reported by these study groups. Although this field of research is recent, controversial results exist. Some in vitro studies on MSCs from different species have shown that under appropriate conditions and with proper inducers, MSCs had the capability to differentiate into male GCs $(13,18-24)$. Although a number of researchers believe that MSCs do not impact regeneration of testicular gametogenesis in infertile animal models (25, 26), other studies have shown that MSCs positively affect reconstruction of germinal epithelium and restoration of fertility (27-30).

In the current study, we evaluated the effects of autologous BM-MSCs on regeneration of testicular epithelium and spermatogenesis recovery by their transplantation into the testes of busulfan-induced infertile male rats at various time points. After aspiration of BM samples, we induced infertility in the rats by administration of a single injection of $40 \mathrm{mg} / \mathrm{kg}$ body weight (BW) busulfan solution. The rat BM-MSCs were isolated and characterized. P3 to 5 cells were labeled with PKH26 and transplanted back into the testes of the animals from which they were derived. The testes were histologically assessed at 4, 6 and 8 weeks after transplantation. We assessed cell viability in the seminiferous tubules, homing and differentiation into the spermatogenic and Sertoli cells (SCs), colony formation, involvement in spermatogenesis and migration into other organs of the body such as the lungs, BM, spleen, and liver. Results were compared between three groups. Any of the previously stated studies used autologous stem cells for transplantation and none of them also reported quantitative information about the fate of transplanted MSCs (25, 27-29). Moreover, in any of them the fate of transplanted cells was assessed and compared in different periods of time after transplantation. The results of this research could assist with the establishment of an appropriate cell-based method of therapy for male infertility.

\section{Materials and Methods}

\section{Study design}

We evaluated the effects of BM-MSCs on germinal epithelium of seminiferous tubules from busulfan-induced infertile male rats. Initially, MSCs were isolated from the BM samples of male rats' tibias. After 10 14 days, each rat received a single dose of busulfan $(40 \mathrm{mg} / \mathrm{kg} \mathrm{BW})$ for induction of infertility. After approximately 28 days, passages-3 (P3) to 5 (P5) BM-MSCs from each male rat were labeled with PKH26 dye (Sigma, USA). Next, we per- 
formed autologous cell transplantations. The rats were divided into three treatment groups. In the first group testicular evaluations were performed 4 weeks after transplantation. In the second group evaluations were performed 6 weeks after transplantation and in the third group evaluations were performed 8 weeks after transplantation.

\section{Animals}

Male Wistar rats that weighed $250 \pm 20 \mathrm{~g}$ were used in this study. Animals were kept under controlled temperature $\left(20^{\circ} \mathrm{C}\right)$ and a 12-h light/12-h dark cycle with ad libitum access to food and water. All procedures were performed following official permission granted by the Animal Ethics Committee of the Faculty of Veterinary Medicine, Urmia University.

\section{Bone marrow (BM) sampling and isolation of $\mathrm{BM}$ mesenchymal stem cells (BM-MSCs)}

BM sampling and isolation of BM-MSCs were performed according to a new protocol reported in our recently published paper (31). Briefly, after induction of anesthesia in the rats (with an intraperitoneal injection of $60 \mathrm{mg} / \mathrm{kg}$ ketamine and $6 \mathrm{mg} / \mathrm{kg}$ xylazine) and dorsal recumbency, the knee region was shaved carefully and disinfected using 70\% ethanol and povidone iodine. A 22-g needle was slowly inserted through the skin on the anterior face of the proximal end of the tibia bone. The needle was penetrated into the bone lumen by revolving movements. Then, the BM was aspirated gently with a syringe that contained $0.1 \mathrm{ml}$ heparin sulfate. All BM samples were immediately mixed with $1 \mathrm{ml}$ complete culture medium [high-glucose Dulbecco's modified eagle medium (DMEM; Gibco, Paisley, UK), 15\% fetal bovine serum (FBS; Gibco, Paisley, UK), $100 \mathrm{U} / \mathrm{ml}$ penicillin G, and $100 \mathrm{U} / \mathrm{ml}$ streptomycin (Gibco, Paisley, UK)].BM/medium mixture was centrifuged (1200 rpm for $5 \mathrm{~min}$ ) and after removal of the supernatant, the cellular pellet was resuspended in fresh warm culture medium in $25 \mathrm{~cm}^{2}$ cell culture flasks and transferred to an incubator $\left(37^{\circ} \mathrm{C}\right.$ and humidified $5 \% \mathrm{CO}_{2}$ atmosphere). The medium was changed every $3 \sim 4$ days and the cells were sub-cultured prior to complete confluency.

\section{Characterization of bone marrow mesenchymal stem cells (BM-MSCs)}

According to criteria suggested by the International Society for Cellular Therapy (32), characterization of rat BM-MSCs was performed based on behavioral characteristics of the cells (adhesion of cells to the bottom of a plas- tic culture dish), expression of specific CD-markers (flow cytometric analysis) and the trilineage (bone, fat and cartilage) differentiation test. Expressions of CD44, CD90, CD11b and CD34 were assessed in P3 cells with specific phycoerythrin (PE) - or fluorescein isothiocyanate (FITC)-conjugated antibodies (Pharmingen BD, San Diego, CA).

For induction of differentiation into bone, fat and cartilage, P3 BM isolated cells were treated with osteogenic, adipogenic and chondrogenic media which was the same culture media used in our previous study (31) for 21 days. Generation of osteocytes, adipocytes and chondrocytes was confirmed by specific staining (alizarin red for osteogenic, oil red $\mathrm{O}$ for adipogenic and toluidine blue for chondrogenic differentiation), reverse transcription polymerase chain reaction (RT-PCR) and detection of the expressions of bone, fat and cartilage specific genes in the cells after 21 days treatment.

\section{RNA isolation and reverse transcription polymerase chain reaction (RT-PCR)}

RT-PCR was utilized to detect the expressions of bone, cartilage and fat specific genes after treatment of BM-MSCs with osteogenic, chondrogenic, and adipogenic media. The RT-PCR process was performed according to former reports $(33,34)$. Briefly, TRIzol reagent (Invitrogen, Paisley, $\mathrm{UK}$ ) was used to extract total RNA from the cells according to the manufacturer's instructions. The extracted RNA underwent DNase treatment by DNase I (Takara, Shiga, Japan) for elimination of genomic DNA. Using random hexamer primer and the RevertAid ${ }^{\mathrm{TM}}$ First Strand cDNA Synthesis Kit (Fermentas, St. Leon-Roth, Germany), $2 \mu \mathrm{g}$ of total RNA was reverse transcribed into cDNA. We used $2 \mu 1$ of produced cDNA together with $1 \times$ PCR buffer (AMSTM; CinnaGen Co., Tehran, Iran); $200 \mu \mathrm{M}$ dNTPs; $0.5 \mu \mathrm{M}$ each of bone, fat and cartilage tissue specific forward and reverse primers; and $1 \mathrm{U}$ Taq DNA polymerase (CinnaGen Co., Tehran, Iran) for the PCR reaction. The primers used for detection of the tissue specific genes in this study are shown in Table 1 .

\section{Preparation of recipient rats}

A single intraperitoneal injection of busulfan $(40 \mathrm{mg} / \mathrm{kg}$ BW, Sigma, USA) was used to induce infertility in the male rats. We confirmed the effects of busulfan on spermatogenesis and induction of infertility in a pilot study before beginning the main study. In this pilot study, we determined the effects of busulfan and its solvent dimethyl sulfoxide (DMSO, Sigma, USA) on testis histology and on sperm count in the vas deferens of the rats. We 
Table 1. Primers used for RT-PCR

\begin{tabular}{llll}
\hline \multicolumn{1}{c}{ Gene } & Sequence A. N. ${ }^{*}$ & \multicolumn{1}{c}{ Forward primer } & Reverse primer \\
B2M & NM_012512.2 & 5'-CACAGTCAAGGCAGAGAAC & 5'-TTCACGCCCATCACAAAC \\
Runx2 & NM_001278484.1 & 5'-ACCCAGGCGTATTCAGATG & 5'-TATGGAGTGCTGCTGGTCTG \\
Osteopontin & AB001382.1 & 5'-GAGGAGAAGGCGCATTACAG & 5'-GTCATCGTCGTCGTCATCAT \\
Aggrecan & NM_022190.1 & 5'-GGGAAGGTTGCTATGGTGAC & 5'-CTCCTGGAAGGTGAATTC \\
Collagen II & NM_012929.1 & 5'-CCAGAACATCACCTACCACT & 5'-CAATGTCAACAATGGGAAGG \\
LPL & NM_012598.2 & 5'-CCCTAAGGACCCCTGAAGAC & 5'-GCCCGATACAACCAGTCTA \\
PPAR $\gamma$ & NM_001145366.1 & 5'-ACCACGGTTGATTTCTCCAG & 5'-GCTTTATCCCCACAGACTCG \\
\hline
\end{tabular}

*A.N.: Accession number.

used $1 \mathrm{~cm}$ of the distal end of the vas deferens to determine the sperm count by a hemocytometer.

\section{PKH26 labeling procedure}

Previous reports have shown that PKH26 is an efficient and reliable dye for tracking of donor cells in the testis $(35,36)$, so, here we selected this dye for labeling of the BM-MSCs before transplantation into the testes. Labeling with PKH26 (Sigma, USA) was performed according to the manufacturer's instructions. Briefly, after detachment of the BM-MSCs by trypsin/EDTA, the cells were counted and washed with medium and no serum. The supernatant was completely removed and immediately we added $1 \mathrm{ml}$ of fresh dye solution that consisted of $2 \mu \mathrm{mol}$ of PKH26 in $1 \mathrm{ml}$ of PBS to each of the $10 \times 10^{6}$ cells, after which the cells were resuspended by pipetting. The cell/dye mixture was incubated for $4 \mathrm{~min}$. An equal volume of FBS $(1 \mathrm{ml})$ was added to the suspension to block the staining process. After $1 \mathrm{~min}$, the suspension was centrifuged at $1200 \mathrm{rpm}$ for $10 \mathrm{~min}$ and the supernatant was cautiously removed. We washed the cells three times with $5 \mathrm{ml} \mathrm{com-}$ plete culture medium and after checking for viability, they were resuspended in $1 \mathrm{ml}$ complete culture medium and placed on ice until transplantation.

\section{Transplantation of labeled bone marrow mesenchymal stem cells (BM-MSCs) into the testis}

PKH26-labeled cells were transplanted into the testes of infertile recipient rats by a method suggested by Ogawa and colleagues (37). There were three study groups in this research- 4 weeks, 6 weeks and 8 weeks. Each treatment group consisted of 6 male Wistar rats. In 5 rats, the left testis was considered as the non-injected control group whereas the right testis received BM-MSCs. In one rat, the left testis was not manipulated, while the other testis received culture medium without cells (medium only or sham group). For transplantation, the recipient rats were anesthetized according to the same method used for BM sampling. The testis was removed from the body cavity through an incision perpendicular to the midline at the upper region of the scrotum. The cell suspension at a concentration of $8-10 \times 10^{6}$ cells $/ \mathrm{ml}$ was transplanted into the rete testis via a sharp thin pipette (each testis received about $2.5 \times 10^{5}$ labeled cells). For better monitoring of the transplantation procedure and observing movement of the cell suspension, a small amount of trypan blue was added to the injection medium. At the end of transplantation, the testis was returned to the body and the muscular layer and skin of the incised region were separately sutured.

\section{Histological evaluations and immunohistochemical staining of the recipient testes}

The recipient testes were evaluated at 4, 6 and 8 weeks after transplantation of BM-MSCs. For this purpose the rats were euthanatized by $\mathrm{CO}_{2}$ inhalation. The testes were removed and some of the seminiferous tubules were observed directly under fluorescent microscope (Olympus, Japan) in order to check the existence of PKH-positive transplanted cells. Then, the testes were placed in Bouin's solution followed by $4 \%$ paraformaldehyde and processed to produce paraffin sections by standard procedures. The $5 \mu \mathrm{m}$ sections were immunostained for Dazl and Stella (markers of GCs) and vimentin (a marker of SCs) and observed for the existence of PKH-positive cells (red fluorescent) that expressed other stained markers. After treatment of the sections with $3 \% \mathrm{H}_{2} \mathrm{O}_{2}$ in distilled water for $30 \mathrm{~min}$ to eliminate endogenous peroxidase, the sections were washed twice in PBS for 5 min each time. Antigen retrieval was performed by boiling the sections in citrate buffer for 8 $10 \mathrm{~min}$ in a microwave followed by washing twice with PBS/Tween (10 min each time). Next, the sections were placed in PBS with 5\% goat serum (PBS-GS) for 1 hour at $37^{\circ} \mathrm{C}$ for blocking and then washed twice with PBS/Tween (5 min each time). Primary antibodies were rabbit polyclonal to Dazl (unconjugated, Abcam, Cambridge, MA, USA, 1/200 in PBS-GS), anti-Stella rab- 
bit polyclonal IgG (unconjugated, Santa Cruz, CA, USA, $1 / 200$ in PBS-GS) and mouse monoclonal to vimentin (FITC-conjugated, Abcam, Cambridge, MA, USA, 1/200 in PBS-GS). The slides were exposed overnight to primary antibodies at $2 \sim 8^{\circ} \mathrm{C}$. Secondary antibodies were goat anti-rabbit IgG (Alexa Fluor ${ }^{\circledR}$ 488-conjugated, Abcam, Cambridge, MA, USA, 1/200 in PBS-GS) for Dazl and goat anti-rabbit IgG (FITC-conjugated, Santa Cruz, CA, USA, 1/100 in PBS-GS) for Stella, which were incubated with the slides for 1 hour at room temperature. After washing three times with PBS/Tween (5 min each time), the cells' nuclei were stained with DAPI (Vector Laboratories, Burlington, ON, Canada) and slides were assessed under a fluorescent microscope (Olympus, Japan). For each group, we determined the following items: existence of PKH-positive cells; percentage of tubules that contained transplanted cells; percentage of tubules positive for donor cells in their germinal epithelium (homed cells); numbers of transplanted BM-MSC-derived colonies and their ratio to the numbers of transplanted cells; percentage of tubules that contained homed transplanted BM-MSCs in their germinal epithelium that also expressed GC- or SC-specific markers; differentiation of transplanted cells into spermatogonia (any type), spermatocytes, spermatids or spermatozoa; calculation of the Tubular Differentiation Index (TDI) which is defined as the percentage of tubules that reach a particular stage of development (having at least three GCs at a specific stage) (38); existence of any transplanted cell-derived sperm (PKH-positive sperm) in the epididymal contents; and finally the existence of transplanted cells in the lungs, BM, spleen and liver (probable migration from the testes after transplantation).

\section{Statistical analysis}

One-way ANOVA and Tukey's HSD were used for data analysis. Version 18 SPSS software (SPSS Inc., Chicago, IL, USA) was used for analysis. $\mathrm{p}<0.05$ was considered to be statistically significant.

\section{Results}

\section{Characterization of bone marrow mesenchymal stem cells (BM-MSCs)}

Three main characteristics of MSCs- adhesion onto the bottom of culture flasks, expression of specific CD markers and potency of differentiation into osteocytes, adipocytes and chondrocytes were checked in the isolated cells obtained from the BM samples. The isolated cells were adhesive cells that had fibroblast-like morphology in the culture. Flow cytometric analysis showed that isolated cells were highly positive for CD44 and CD90 and negative for CD11b and CD34 (Fig. 1). The trilineage differentiation test showed that after 21 days treatment of BM-MSCs with osteogenic, adipogenic and chondrogenic media, the cells differentiated into osteocytes, adipocytes and chondrocytes, respectively. Differentiation was confirmed by specific staining, RT-PCR, and detection of expressions of bone, fat and cartilage-specific genes in the treated cells (Fig. 2).

\section{Preparation of recipient rats}

We performed a pilot study to confirm the efficacy of
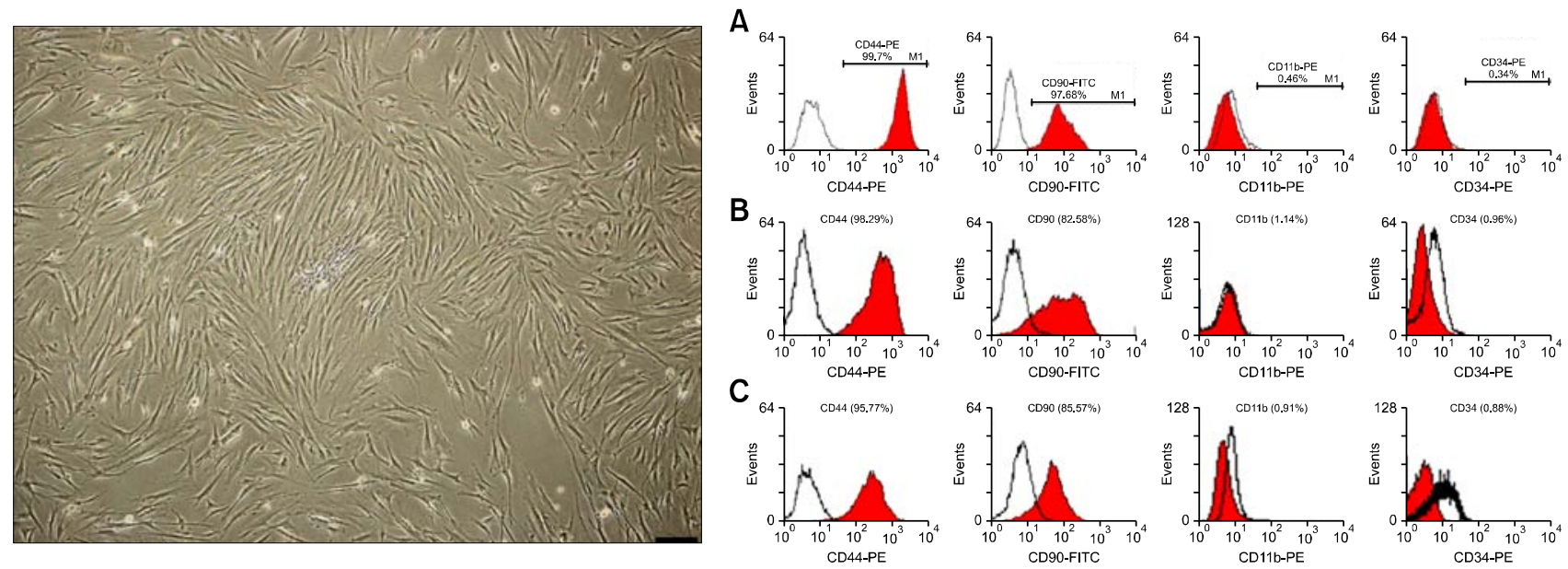

Fig. 1. Left: rat elongated fibroblast-like bone marrow mesenchymal stem cells (BM-MSCs) $(B a r=200 \mu \mathrm{m})$. Right: flow cytometric analysis of rat bone marrow mesenchymal stem cells (BM-MSCs) surface CD markers. The cells of 4-week (A), 6-week (B) and 8-week (C) groups were highly positive for CD44 and CD90 and almost negative for CD11b and CD34. 
A

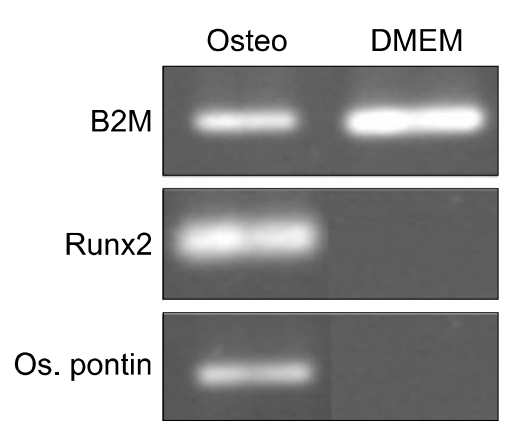

B

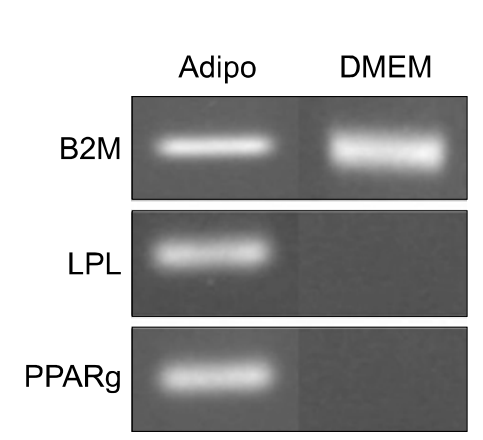

C

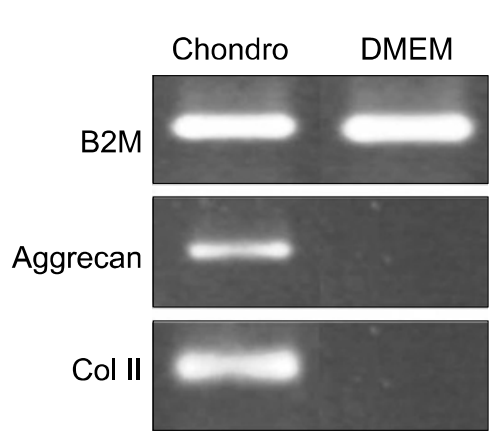

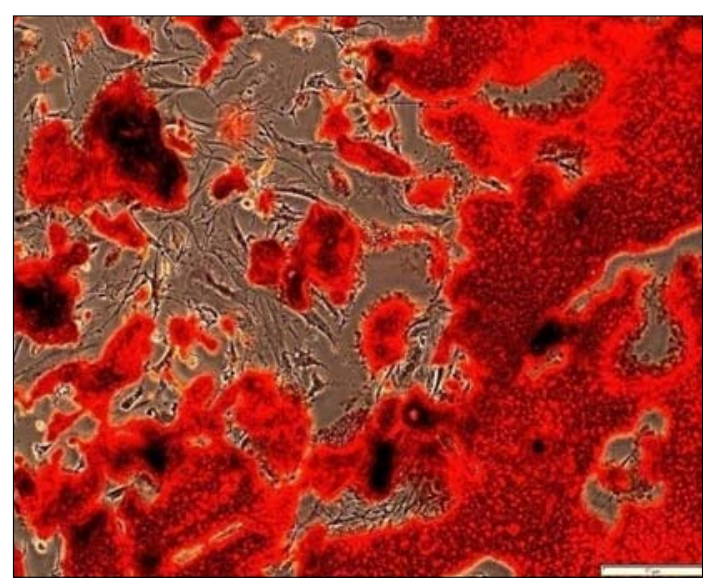
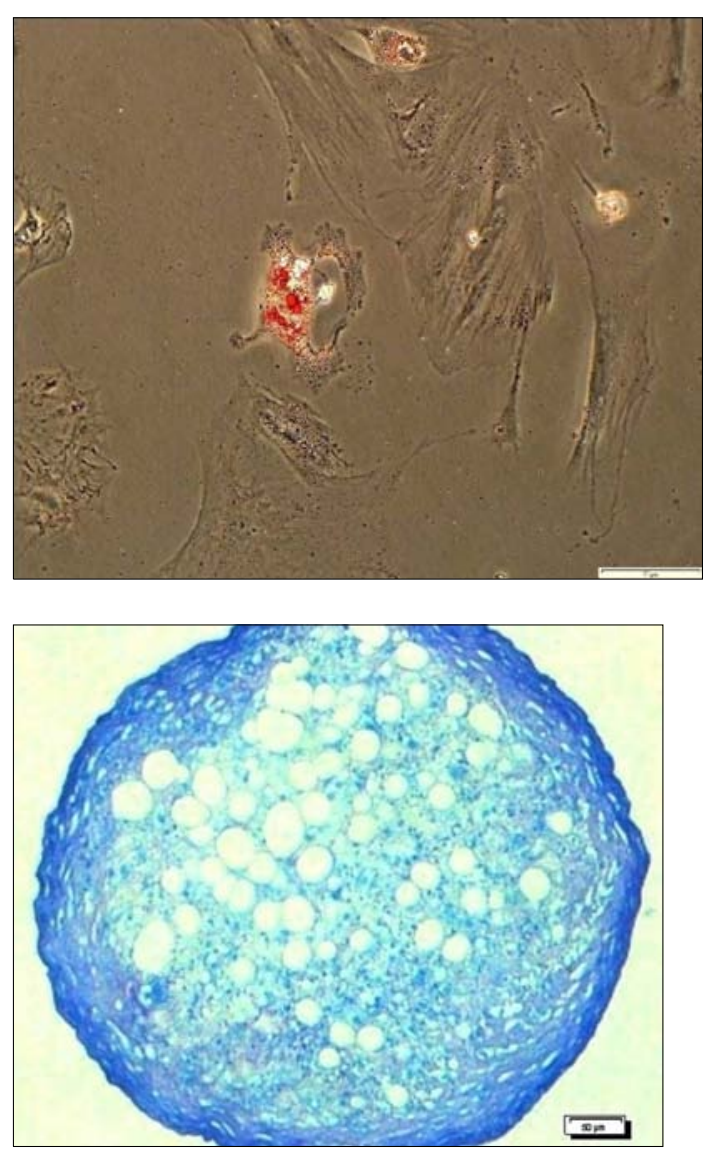

Fig. 2. Trilineage differentiation test of rat bone marrow mesenchymal stem cells (BM-MSCs). Differentiation was confirmed with specific staining and evaluation of tissue-specific gene expressions. (A) Osteogenic differentiation. (B) Adipogenic differentiation. (C) Chondrogenic differentiation (Bars $=50 \mu \mathrm{m})$. busulfan for induction of infertility in male rats. Histological evaluations indicated that a single injection of $40 \mathrm{mg} / \mathrm{kg}$ BW busulfan in the rats completely destroyed the germinal epithelium of the seminiferous tubules. We observed that DMSO (busulfan solvent) had no negative impact on the germinal layer. Busulfan effectively disturbed spermatogenesis and significantly decreased the sperm count (Fig. 3).

\section{Cell transplantation procedure}

PKH-labeled BM-MSCs were transplanted into each testis through the rete testis by a thin pipette. The addition of trypan blue enabled us to monitor the medium flow in the seminiferous tubules (Fig. 4D).

\section{Histological evaluations and fate of bone marrow mesenchymal stem cells (BM-MSCs) in the testis}

We removed the testes at 4, 6 and 8 weeks after transplantation in order to determine the fate of transplanted 

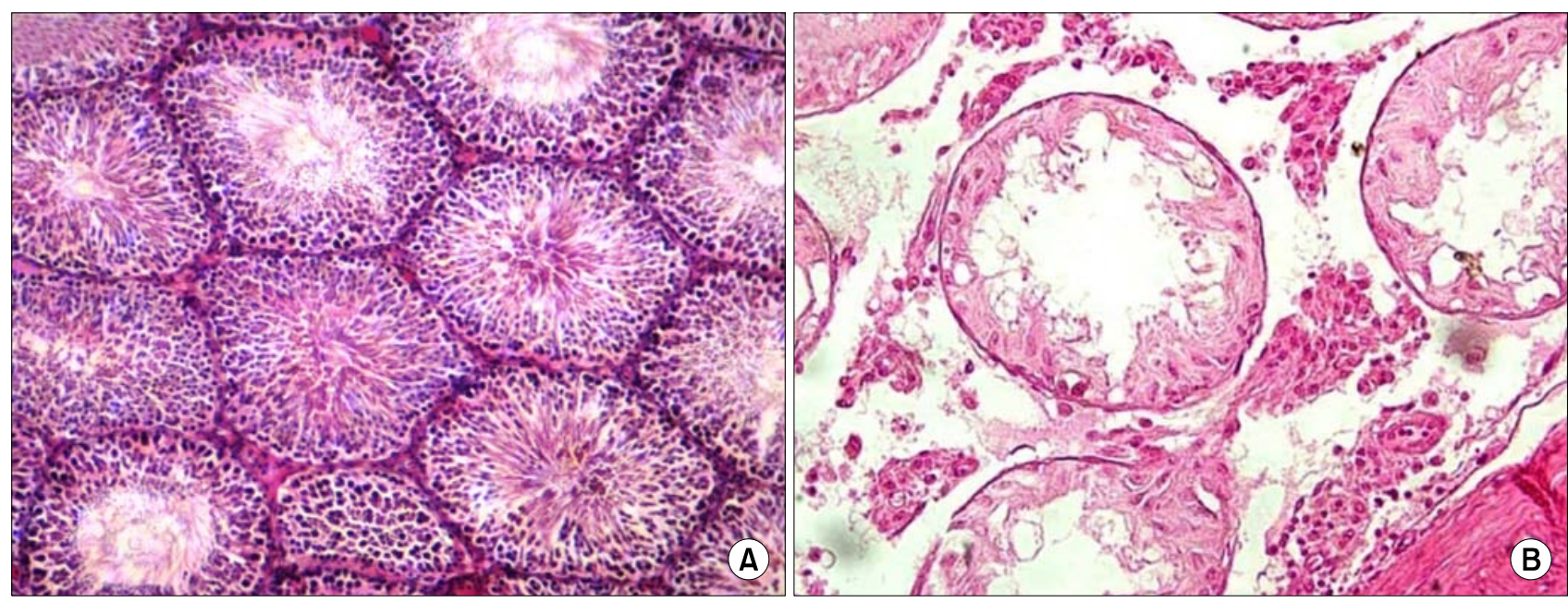

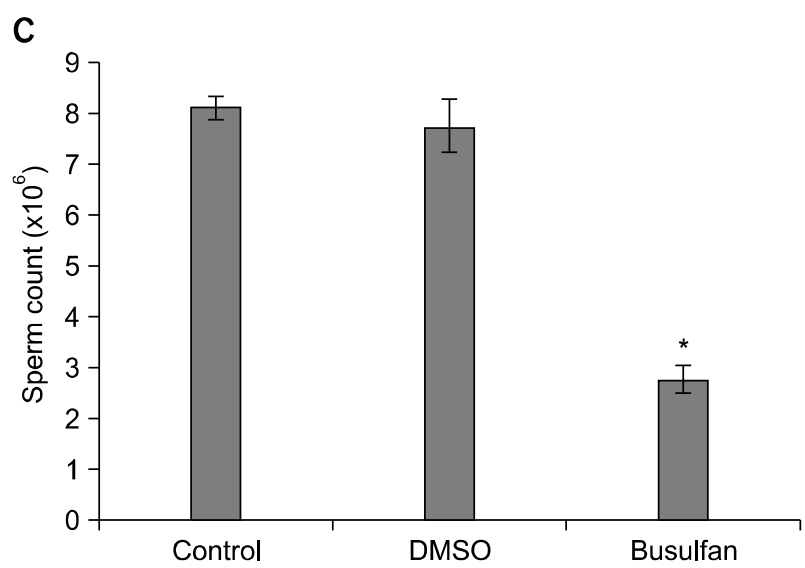

BM-MSCs in the testes at different times after transplantation. Any sign of tumor formation or infection was recorded in the testes of the different study groups.

\section{Existence of transplanted bone marrow mesenchymal stem cells (BM-MSCs) in the testes}

Direct observation of seminiferous tubules from all three study groups showed the presence of transplanted BM-MSCs in the testes at 4, 6 and 8 weeks post-transplantation (Fig. 4A and B).

\section{Percentage of seminiferous tubules that contained transplanted cells}

Histological evaluations showed that although the transplanted cells were alive in the testes of all three treatment groups, the mean percentage of seminiferous tubules that contained donor cells differed among the three groups. This percentage was approximately $12.2 \%$ for 4-week, $4.8 \%$ for 6-week and $1.2 \%$ for 8 -week testes (Table 2). Comparison of the results showed that the percentage of seminiferous tubules that contained transplanted cells de-
Fig. 3. Effects of busulfan on testicular tissue. (A) Normal testis. (B) Busulfan treated testis. (C) Sperm counts in normal, dimethyl sulfoxide (DMSO; solvent of busulfan) and busulfan treated rats. *Significant difference $(p<0.05)$.

creased significantly with increasing time (Fig. 4C).

\section{Homing of the transplanted cells}

Evaluation of histological sections from the testes of the three treatment groups revealed that some of the transplanted PKH-positive BM-MSCs located or homed at the germinal epithelium on the basement membrane of the seminiferous tubules. The mean percentage of tubules that contained these homed cells was $0.16 \%$ for 4 -week, $0.072 \%$ for 6-week and $0.008 \%$ for 8-week testes (Table 2). Immunohistochemistry showed that most of these homed cells expressed GC-specific markers, Stella and Dazl (Figs. 5 and 6). Fig. 5E shows a comparison of the mean percentages of homed cell-containing tubules in the three treatment groups.

\section{Transplanted cell-derived colony}

Among all study group testes, only one testis from the 4-week group contained a cell colony-like compartment that originated from the transplanted $\mathrm{PKH}$-positive cells (Table 2). Fig. 6 shows atransplanted PKH-positive 

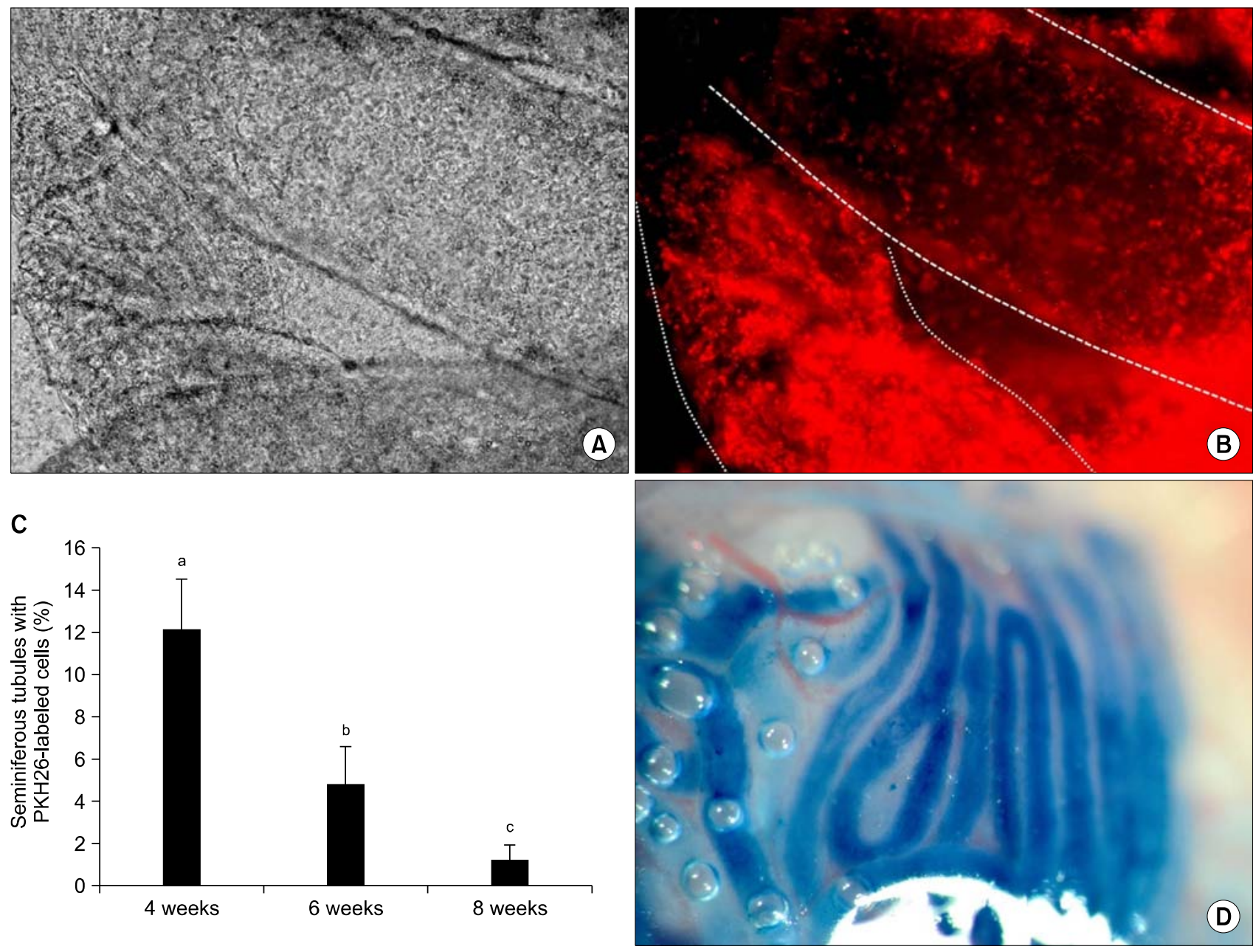

Fig. 4. Existence of PKH-labeled cells in the seminiferous tubules of a testis 8 weeks after transplantation. (A) Tow seminferous tubules under white light, (B) the same tubules under fluorescent light. (C) Percentage of tubules that contained transplanted cells in different study groups. The percentage of positive tubules decreased over time. Different letters represent significant difference between groups $(p<0.05)$. (D) Filling the seminiferous tubules by the cell suspension that contained trypan blue.

Table 2. Data obtained after evaluation of testes from different study groups

\begin{tabular}{|c|c|c|c|c|c|c|c|c|c|}
\hline \multirow{2}{*}{ Groups } & \multirow{2}{*}{$\begin{array}{l}\text { Tumor formation, } \\
\text { infection or } \\
\text { inflammation }\end{array}$} & \multirow{2}{*}{$\begin{array}{c}\text { Seminiferous } \\
\text { tubules contained } \\
\text { labeled cells }(\%)\end{array}$} & \multirow{2}{*}{$\begin{array}{c}\text { Seminiferous } \\
\text { tubules contained } \\
\text { homed cells }(\%)\end{array}$} & \multirow{2}{*}{ Colony number } & \multicolumn{5}{|c|}{ Tubular Differentiation Index (TDI)* } \\
\hline & & & & & Spg. & Spc. & Spt. & Sp. & SC \\
\hline $\begin{array}{l}\text { Unmanipulated } \\
\text { control }\end{array}$ & - & 0 & 0 & 0 & 0 & 0 & 0 & 0 & 0 \\
\hline $\begin{array}{l}\text { Culture } \\
\text { medium only }\end{array}$ & - & 0 & 0 & 0 & 0 & 0 & 0 & 0 & 0 \\
\hline 4 weeks & - & $12.2^{\mathrm{a}}$ & $0.16^{\mathrm{a}}$ & $1 * *$ & $0.14^{\mathrm{a}}$ & 0 & 0 & 0 & 0 \\
\hline 6 weeks & - & $4.8^{\mathrm{b}}$ & $0.072^{\mathrm{b}}$ & 0 & $0.05^{\mathrm{b}}$ & 0 & 0 & 0 & 0 \\
\hline 8 weeks & - & $1.2^{\mathrm{c}}$ & $0.008^{\mathrm{C}}$ & 0 & $0.0068^{\mathrm{c}}$ & 0 & 0 & 0 & 0 \\
\hline
\end{tabular}

*TDI is defined as the percentage of tubules reaching a particular stage of development (having at least three germ cells at a specific stage) (38).

**Just one colony-like compartment was observed in a testis of 4 week group.

-Different letters represent significant difference between the amount of the treatment groups in a column $(p<0.05)$. 

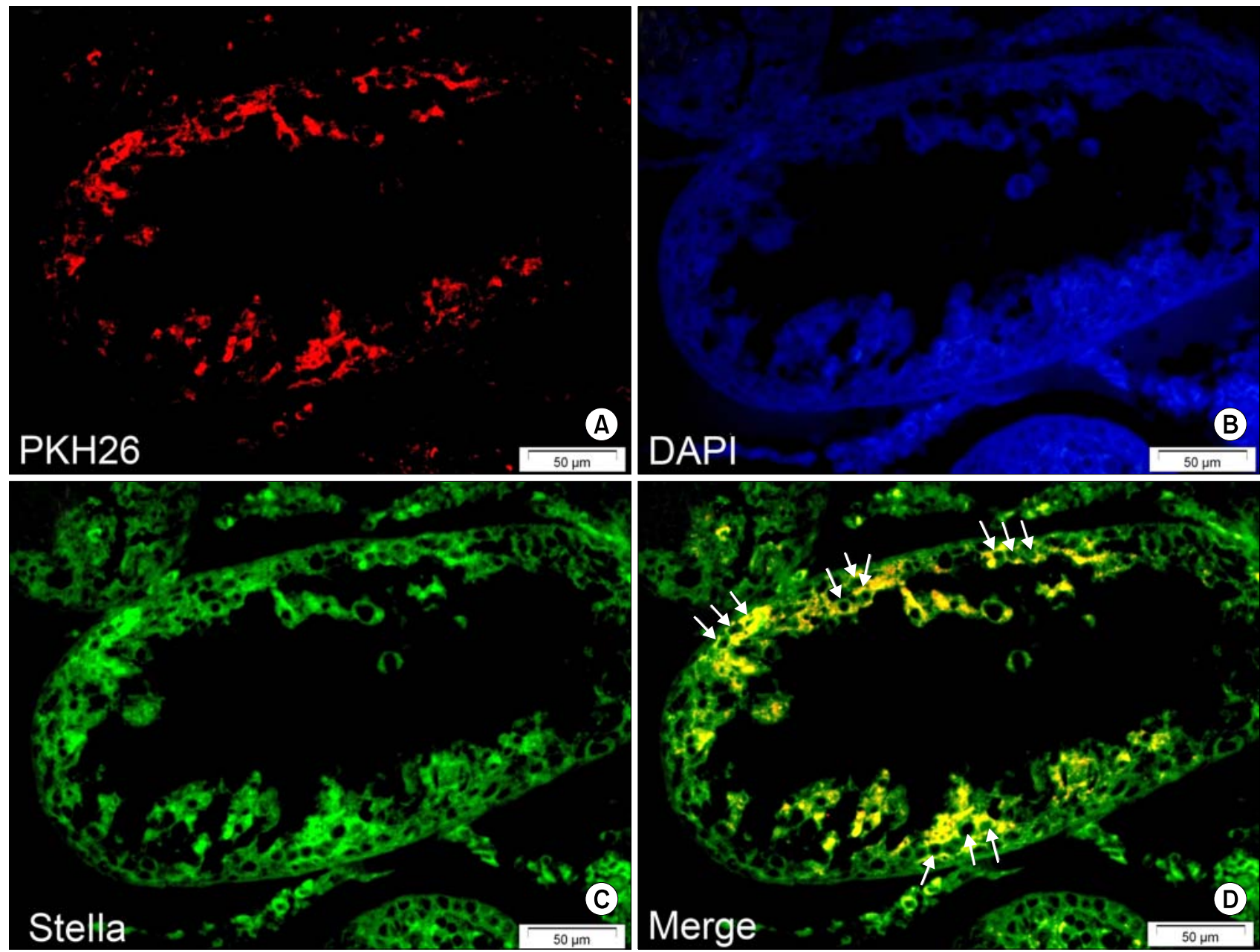

E

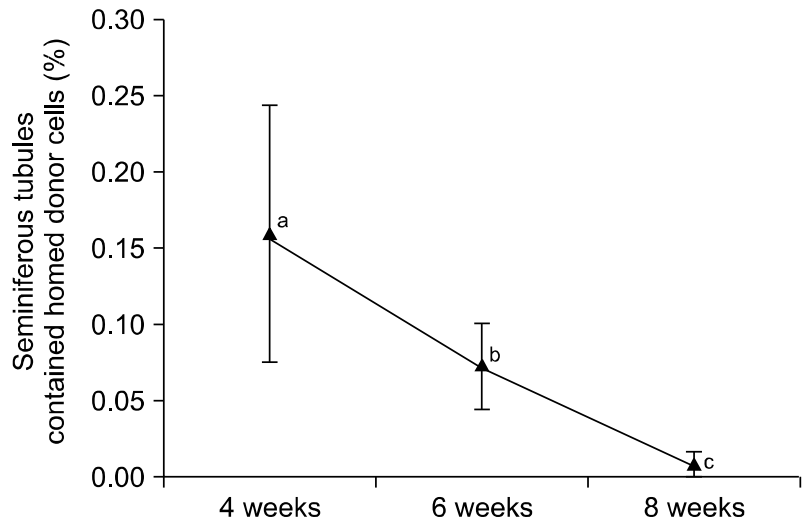

Fig. 5. Homed PKH-positive bone marrow mesenchymal stem cells (BM-MSCs) express a germ cell-specific marker (Stella) at the germinal epithelium of seminiferous tubules. (A) PKH26. (B) DAPI. (C) Stella (FITC). (D) Merged picture. In the merged picture arrows show a number of spermatogonia-like cells that express Stella. (E) The percentage of tubules that contained homed cells in the different groups. As can be seen, the percentage of positive seminiferous tubules decreased with time progression. Different letters represent significant difference between groups $(p<0.05)$ (Bars $=50 \mu \mathrm{m}$ ).

cell-derived cell mass together with a number of homed cells that expressed the GC marker Dazl.

\section{Differentiation of transplanted bone marrow} mesenchymal stem cells (BM-MSCs) into germ cells (GCs) and Sertoli cells (SCs)

Testes tissue sections were immunostained for two GC

markers (Dazl and Stella) and a SC marker (vimentin). Evaluations indicated that most transplanted cells that homed at the germinal epithelium of the seminiferous tubules expressed Dazl and/or Stella (Figs. 5 and 6). This finding confirmed the differentiation of BM-MSCs into male spermatogonia-like GCs. In addition, we measured TDI for different stages of GC development (spermatogonia, 


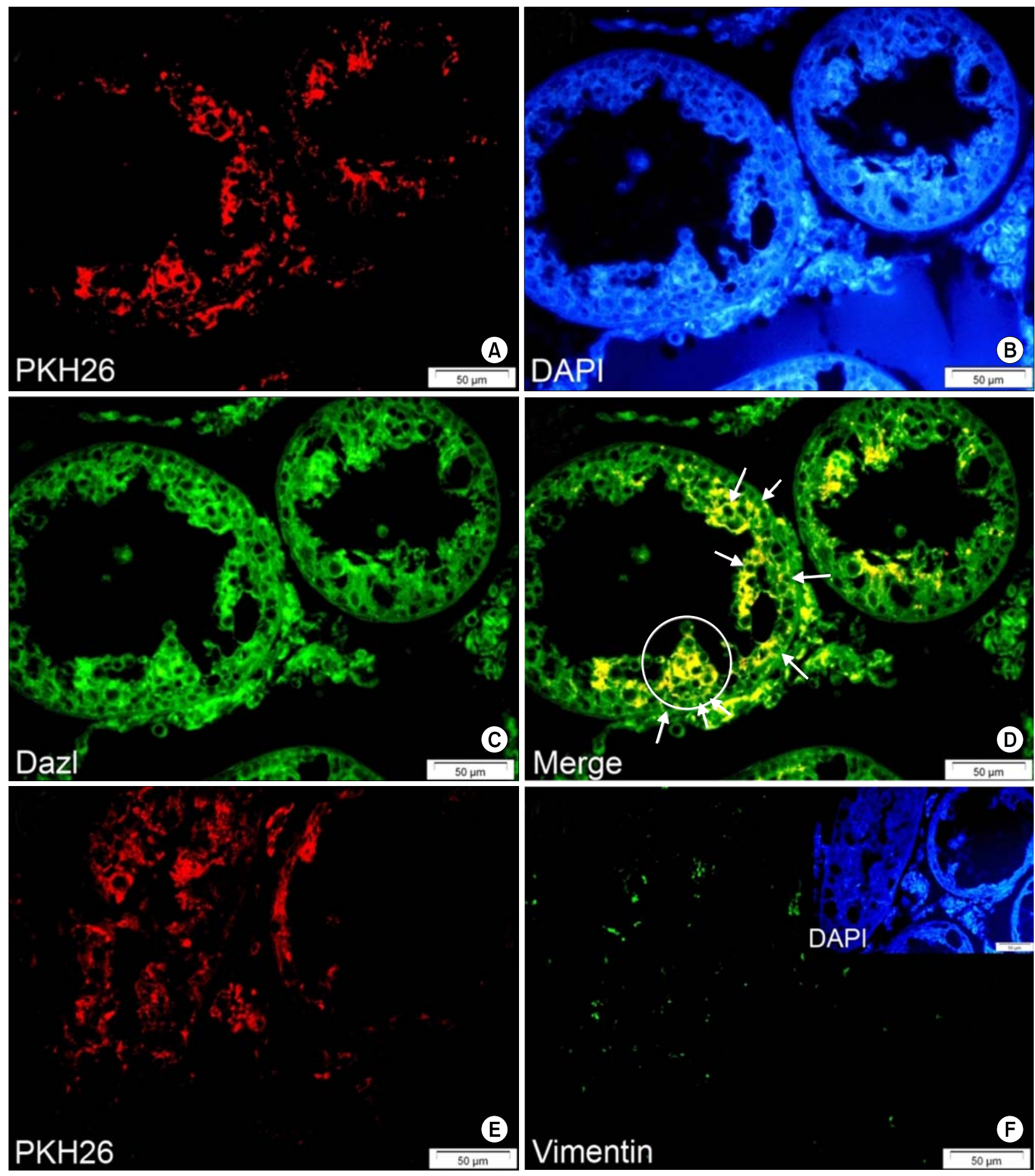

Fig. 6. Only one transplanted cell-derived colony-shaped compartment was observed in all evaluated testes. (A) PKH26. (B) DAPI. (C) Dazl (FITC). (D) Merged picture. The circle shows the colony-like cell aggregate of PKH-positive transplanted cells that simultaneously expressed Dazl. Arrows show spermatogonia-like cells that express both PKH26 and the germ cell-specific marker, Dazl. PKH26-labeled BM-MSCs in the seminiferous tubules of recipient testis did not express sertoli cell-specific marker, after 4, 6 and 8 weeks. (E) PKH26 and (F) Vimentin (FITC) (Bars $=50 \mu \mathrm{m})$. 
spermatocytes, spermatids and sperm). TDI for spermatogonia was $0.14 \%$ in 4 -week, $0.05 \%$ in 6-weekand $0.0068 \%$ in 8-week testes. TDI for spermatocytes, spermatids and sperm was 0 in all study groups. We observed no PKH-positive sperm in the epididymal (vas deferens) contents of all three groups. In addition, our results showed that transplanted MSCs did not express vimentin (Fig. 6 $\mathrm{E}$ and F). They did not differentiate into SCs in any of the study groups (Table 2).

\section{Migration of transplanted cells into other organs}

We assessed the lungs, BM, spleen and liver in order to determine if any labeled BM-MSCs migrated into other organs after transplantation into the testis. No PKH-labeled cells were observed in these organs in any of the treatment groups. Therefore no migration occurred after injection of the cells into the testes.

\section{Discussion}

A number of in vitro studies confirmed that MSCs have the capacity to differentiate into GCs (13, 18, 20-24). Transplantation studies on the effects of MSCs on reconstruction of testicular germinal epithelium in infertile male animals, showed a number of promising results. Some studies reported that MSCs had no positive effects on regeneration of germinal epithelium, nor could differentiate into GCs in the testis $(25,26)$. However others reported that transplanted MSCs not only had the potency for differentiation into GCs (28), but also they could fully differentiate into sperm and regenerate spermatogenesis $(27,29)$. A recent study has showed the supportive role of BM-MSCs for recovery of spermatogenesis after transplantation into the testes of busulfan-induced infertile male hamsters (30). In the current study, we evaluated the fate of rat autologous BM-MSCs after transplantation into the testes of busulfan-induced infertile rats at 4, 6 and 8 weeks after transplantation. Any of the previous studies was performed on autologous MSCs.

BM sampling and isolation of BM-MSCs were performed according to our previously published paper (31). Isolated cells were characterized using the criteria proposed by the International Society for Cellular Therapy defined as: adhesion onto the bottom of the culture dish, expression of MSC CD markers, lack of expression of other cell lineage CD markers, and the capacity of differentiation into osteocytes, adipocytes and chondrocytes (32). Our isolated BM cells were elongated adhesive cells that highly expressed CD44 and CD90 with very low expressions of CD11b and CD34 according to flow cyto- metric analysis. After treatment with osteogenic, adipogenic and chondrogenic media, the cells differentiated into osteocytes, adipocytes and chondrocytes, respectively. This differentiation was confirmed by specific staining and RT-PCR analysis and detection of the expressions of tissue-specific genes.

Following isolation of the BM-MSCs, the rats were made infertile by busulfan injection. Our pilot study confirmed that $40 \mathrm{mg} / \mathrm{kg}$ BW busulfan could efficiently disrupt spermatogenesis and destroy the germinal epithelium in male rats at 28 days after a single intraperitoneal injection. After induction of infertility in the male rats, we transplanted their autologous BM-MSCs (P3 to P5) into the rete testis according to the method described by Ogawa (37). The transplanted testes were histologically evaluated at 4, 6 and 8 weeks after transplantation. Existence of the transplanted cells in the seminiferous tubules, their homing, differentiation into GCs and SCs, colony formation, involvement in the spermatogenesis process, and migration into other organs were evaluated and compared in the three groups.

Our assessments revealed that transplanted BM-MSCs remained viable in the 4-, 6- and 8-week testes. The numbers of seminiferous tubules that contained transplanted cells significantly decreased with increased time. The number of positive tubules in the 4-week group were more than the 6-week group $(\mathrm{p}<0.05)$ and in the 6-week group was more than the 8-week group $(\mathrm{p}<0.05)$ (Fig. 4C). Although, no study reported similar quantitative data, in total, controversial reports existed regarding the viability of MSCs after transplantation into testes. A group of researchers have stated that MSCs could not survive in the testis (25), whereas others have shown that MSCs were alive 2 months after transplantation (27-29). This finding showed that, transplanted BM-MSCs decreased (died and absorbed) with progression of time. According to the results from all three treatment groups, a number of transplanted BM-MSCs migrated into the germinal epithelium and homed on the basement membrane of the seminiferous tubules. The mean percentage of the seminiferous tubules that contained homed cells was $1.6 \%$ in the 4-week group, $0.072 \%$ in the 6-week group and $0.008 \%$ in the 8 -week group. Differences between the percentages of three treatment groups were statistically significant $(p<0.05)$. Immunohistochemistry showed that the most of the homed cells expressed GC-specific markers (Dazl or Stella), however their numbers decreased as time progressed (Figs. 5 and 6 and Table 2). There was no quantitative information about the percentage of homing of transplanted cells into the testis, however three studies 
have shown that MSCs could locate in the germinal epithelium and mimic the characteristics of native spermatogonia (27-29).

Some studies showed that male adult GCs or spermatogonial stem cells located at the germinal epithelium and formed colonies after transplantation into the testis (39, 40). In the current study, we observed only one possible colony of transplanted cells formed in the testis of the 4-week group (Fig. 6). No other colonies were observed neither in the testes from the remainder of the 4-week group, nor in the 6- and 8-week groups. There was no report that discussed colony formation of transplanted MSCs in the testis. One study reported that $10 \times 10^{6}$ in vitro generated male GCs from mouse MSCs formed 3 5 colonies in the testes of recipient mice (13).

In this research we calculated the TDI for different stages of testis GCs that included spermatogonia, spermatocytes, spermatids and sperm. As shown in Table 2, TDI for spermatogonia was $0.14 \%$ in the 4 -week group, $0.05 \%$ in the 6-week group and $0.0068 \%$ in the 8-week group. TDI for the other stages of GCs were 0 for all study groups. There was no quantitative report about the differentiation of transplanted MSCs in seminiferous tubules.

Reports regarding the fate of MSCs in the testes of infertile animals are conflicting. A number of researchers have shown that MSCs can differentiate into the spermatogonia and re-establish spermatogenesis after transplantation into the testis (27-29), whereas others suggest that MSCs cannot regenerate testicular germinal epithelium $(25,26)$. Our results have shown that rat autologous BM-MSCs differentiated into the spermatogonia after transplantation into the testis of infertile male rats. This was confirmed by immunohistochemistry and detection of expressions of spermatogonia specific markers Dazl and Stella in homed PKH-positive cells. Their numbers decreased with progression of time and we observed no further differentiation of transplanted cells and donor-derived sperm. This finding has proven the capacity of MSCs to differentiate into spermatogonia in the testes. These promising findings will be a proper basis for establishing an appropriate in vitro or in vivo system for GC production from stem cells. Possibly by optimization of the MSC culture conditions prior to transplantation by the use of some inducers (retinoic acid) or growth factors such as glial cell line-derived neurotrophic factor (GDNF) complete spermatogenesis may eventually be achieved and solve the problem of infertility. Additional detailed studies should be performed to answer several questions in the field of spermatogenesis and treatment of infertility with stem cell-based therapies. With such studies, and also modification of the culture and treatment systems, certainly, regeneration of damaged testicular germinal epithelium of infertile males and restoration of spermatogenesis using autologous MSCs, do not seem so out of reach.

\section{Acknowledgments}

We would like to express our appreciation to Dr. Mohammadali Khalilifar and Miss. Maryam Ghasemzadeh-Hasankolaei for their critical assistance with the cell labeling process. This work was supported financially, by a research grant (number 91002356), from Iran National Science Foundation (INSF) and conducted in collaboration with the Royan institute.

\section{Potential conflict of interest}

The authors have no conflicting financial interest.

\section{Author contributions}

All the authors contributed equally in the design, research work and manuscript preparation.

\section{References}

1. Nayernia K, Li M, Jaroszynski L, Khusainov R, Wulf G, Schwandt I, Korabiowska M, Michelmann HW, Meinhardt A, Engel W. Stem cell based therapeutical approach of male infertility by teratocarcinoma derived germ cells. Hum Mol Genet 2004;13:1451-1460

2. Madhukar D, Rajender S. Hormonal treatment of male infertility: promises and pitfalls. J Androl 2009;30:95-112

3. Miyamoto T, Tsujimura A, Miyagawa Y, Koh E, Namiki $M$, Sengoku K. Male infertility and its causes in human. Adv Urol 2012;2012:384520

4. Esteves SC, Miyaoka R, Agarwal A. Surgical treatment of male infertility in the era of intracytoplasmic sperm injection - new insights. Clinics (Sao Paulo) 2011;66:1463-1478

5. Volarevic V, Bojic S, Nurkovic J, Volarevic A, Ljujic B, Arsenijevic N, Lako M, Stojkovic M. Stem cells as new agents for the treatment of infertility: current and future perspectives and challenges. Biomed Res Int 2014;2014: 507234

6. Uccelli A, Moretta L, Pistoia V. Mesenchymal stem cells in health and disease. Nat Rev Immunol 2008;8:726-736

7. da Silva Meirelles L, Chagastelles PC, Nardi NB. Mesenchymal stem cells reside in virtually all post-natal organs and tissues. J Cell Sci 2006;119:2204-2213

8. Pountos I, Corscadden D, Emery P, Giannoudis PV. Mesenchymal stem cell tissue engineering: techniques for isolation, expansion and application. Injury 2007;38 Suppl 4:S23-S33

9. Bianco P, Riminucci M, Gronthos S, Robey PG. Bone marrow stromal stem cells: nature, biology, and potential 
applications. Stem Cells 2001;19:180-192

10. Colter DC, Sekiya I, Prockop DJ. Identification of a subpopulation of rapidly self-renewing and multipotential adult stem cells in colonies of human marrow stromal cells. Proc Natl Acad Sci USA 2001;98:7841-7845

11. Nombela-Arrieta C, Ritz J, Silberstein LE. The elusive nature and function of mesenchymal stem cells. Nat Rev Mol Cell Biol 2011;12:126-131

12. Song L, Tuan RS. Transdifferentiation potential of human mesenchymal stem cells derived from bone marrow. FASEB J 2004;18:980-982

13. Nayernia K, Lee JH, Drusenheimer N, Nolte J, Wulf G, Dressel R, Gromoll J, Engel W. Derivation of male germ cells from bone marrow stem cells. Lab Invest 2006; 86:654-663

14. Rojas M, Xu J, Woods CR, Mora AL, Spears W, Roman J, Brigham KL. Bone marrow-derived mesenchymal stem cells in repair of the injured lung. Am J Respir Cell Mol Biol 2005;33:145-152

15. Chen Y, Shao JZ, Xiang LX, Dong XJ, Zhang GR. Mesenchymal stem cells: a promising candidate in regenerative medicine. Int $\mathrm{J}$ Biochem Cell Biol 2008; 40:815-820

16. Reinders ME, Fibbe WE, Rabelink TJ. Multipotent mesenchymal stromal cell therapy in renal disease and kidney transplantation. Nephrol Dial Transplant 2010;25:17-24

17. Farini A, Sitzia C, Erratico S, Meregalli M, Torrente Y. Clinical applications of mesenchymal stem cells in chronic diseases. Stem Cells Int 2014;2014:306573

18. Drusenheimer N, Wulf G, Nolte J, Lee JH, Dev A, Dressel R, Gromoll J, Schmidtke J, Engel W, Nayernia K. Putative human male germ cells from bone marrow stem cells. Soc Reprod Fertil Suppl 2007;63:69-76

19. Ghasemzadeh-Hasankolaei M, Eslaminejad MB, Batavani R, Sedighi-Gilani M. Comparison of the efficacy of three concentrations of retinoic acid for transdifferentiation induction in sheep marrow-derived mesenchymal stem cells into male germ cells. Andrologia 2014;46:24-35

20. Ghasemzadeh-Hasankolaei M, Sedighi-Gilani MA, Eslaminejad MB. Induction of ram bone marrow mesenchymal stem cells into germ cell lineage using transforming growth factor- $\beta$ superfamily growth factors. Reprod Domest Anim 2014;49:588-598

21. Hua J, Pan S, Yang C, Dong W, Dou Z, Sidhu KS. Derivation of male germ cell-like lineage from human fetal bone marrow stem cells. Reprod Biomed Online 2009; 19:99-105

22. Hua J, Yu H, Dong W, Yang C, Gao Z, Lei A, Sun Y, Pan S, Wu Y, Dou Z. Characterization of mesenchymal stem cells (MSCs) from human fetal lung: potential differentiation of germ cells. Tissue Cell 2009;41:448-455

23. Huang P, Lin LM, Wu XY, Tang QL, Feng XY, Lin GY, Lin X, Wang HW, Huang TH, Ma L. Differentiation of human umbilical cord Wharton's jelly-derived mesenchymal stem cells into germ-like cells in vitro. J Cell Biochem 2010;109:747-754
24. Shirazi R, Zarnani AH, Soleimani M, Abdolvahabi MA, Nayernia K, Ragerdi Kashani I. BMP4 can generate primordial germ cells from bone-marrow-derived pluripotent stem cells. Cell Biol Int 2012;36:1185-1193

25. Horn MM, Paz AH, Duarte ME, Baldo G, Belardinelli MC, Matte U, Lima EO, Passos EP. Germinative testicular cells and bone marrow mononuclear cells transplanted to a rat model of testicular degeneration. Cloning Stem Cells 2008; 10:543-546

26. Lassalle B, Mouthon MA, Riou L, Barroca V, Coureuil M, Boussin F, Testart J, Allemand I, Fouchet P. Bone marrow-derived stem cells do not reconstitute spermatogenesis in vivo. Stem Cells 2008;26:1385-1386

27. Cakici C, Buyrukcu B, Duruksu G, Haliloglu AH, Aksoy A, Isık A, Uludag O, Ustun H, Subası C, Karaoz E. Recovery of fertility in azoospermia rats after injection of adipose-tissue-derived mesenchymal stem cells: the sperm generation. Biomed Res Int 2013;2013:529589

28. Monsefi M, Fereydouni B, Rohani L, Talaei T. Mesenchymal stem cells repair germinal cells of seminiferous tubules of sterile rats. Iran J Reprod Med 2013; 11:537-544

29. Zhang D, Liu X, Peng J, He D, Lin T, Zhu J, Li X, Zhang Y, Wei G. Potential spermatogenesis recovery with bone marrow mesenchymal stem cells in an azoospermic rat model. Int J Mol Sci 2014;15:13151-13165

30. Tamadon A, Mehrabani D, Rahmanifar F, Jahromi AR, Panahi M, Zare S, Khodabandeh Z, Jahromi IR, Tanideh $N$, Dianatpour $M$, Ramzi $M$, Koohi-Hoseinabadi O. Induction of Spermatogenesis by Bone Marrow-derived Mesenchymal Stem Cells in Busulfan-induced Azoospermia in Hamster. Int J Stem Cells 2015;8:134-145

31. Ghasemzadeh-Hasankolaei M, Eslaminejad MB, Batavani $\mathrm{R}$, Ghasemzadeh-Hasankolaei $M$. Male and female rat bone marrow-derived mesenchymal stem cells are different in terms of the expression of germ cell specific genes. Anat Sci Int 2015;90:187-196

32. Dominici M, Le Blanc K, Mueller I, Slaper-Cortenbach I, Marini F, Krause D, Deans R, Keating A, Prockop Dj, Horwitz E. Minimal criteria for defining multipotent mesenchymal stromal cells. The International Society for Cellular Therapy position statement. Cytotherapy 2006; 8:315-317

33. Ghasemzadeh-Hasankolaei M, Eslaminejad MB, SedighiGilani M, Mokarizadeh A. Starvation is more efficient than the washing technique for purification of rat Sertoli cells. In Vitro Cell Dev Biol Anim 2014;50:723-730

34. Ghasemzadeh-Hasankolai M, Batavani R, Eslaminejad MB, Sedighi-Gilani $M$. Effect of zinc ions on differentiation of bone marrow-derived mesenchymal stem cells to male germ cells and some germ cell-specific gene expression in rams. Biol Trace Elem Res 2012;150:137-146

35. Ghasemzadeh-Hasankolaei M, Eslaminejad MB, SedighiGilani M. Derivation of male germ cells from ram bone marrow mesenchymal stem cells by three different methods and evaluation of their fate after transplantation into the 
testis. In Vitro Cell Dev Biol Anim 2016;52:49-61

36. Herrid M, Vignarajan S, Davey R, Dobrinski I, Hill JR. Successful transplantation of bovine testicular cells to heterologous recipients. Reproduction 2006;132:617-624

37. Ogawa T, Aréchaga JM, Avarbock MR, Brinster RL. Transplantation of testis germinal cells into mouse seminiferous tubules. Int J Dev Biol 1997;41:111-122

38. Zhang Z, Shao S, Shetty G, Meistrich ML. Donor Sertoli cells transplanted into irradiated rat testes stimulate partial recovery of endogenous spermatogenesis. Reproduction
2009; 137:497-508

39. Rodriguez-Sosa JR, Silvertown JD, Foster RA, Medin JA, Hahnel A. Transduction and transplantation of spermatogonia into the testis of ram lambs through the extra-testicular rete. Reprod Domest Anim 2009;44:612-620 40. Shinohara T, Orwig KE, Avarbock MR, Brinster RL. Remodeling of the postnatal mouse testis is accompanied by dramatic changes in stem cell number and niche accessibility. Proc Natl Acad Sci USA 2001;98:6186-6191 